2016-06-28

\title{
CD147 handles lipid: a new role for anti-cancer target
}

\author{
Felmlee, Daniel
}

http://hdl.handle.net/10026.1/9677

10.21037/tcr.2016.06.28

Translational Cancer Research

AME Publishing Company

All content in PEARL is protected by copyright law. Author manuscripts are made available in accordance with publisher policies. Please cite only the published version using the details provided on the item record or document. In the absence of an open licence (e.g. Creative Commons), permissions for further reuse of content should be sought from the publisher or author. 


\title{
CD147 handles lipid: a new role for anti-cancer target
}

\author{
Daniel J. Felmlee ${ }^{1}$, Thomas F. Baumert ${ }^{2,3,4}$ \\ ${ }^{1}$ Hepatology Research Group, Peninsula School of Medicine and Dentistry, University of Plymouth, Plymouth, UK; ${ }^{2}$ Inserm, U1110, Institut \\ de Recherche sur les Maladies Virales et Hépatiques, Strasbourg, France; ${ }^{3}$ Université de Strasbourg, Strasbourg, France; ${ }^{4}$ Institut Hospitalo- \\ Universitaire, Pôle Hépato-digestif, Hôpitaux Universitaires de Strasbourg, Strasbourg, France \\ Correspondence to: Thomas F. Baumert, MD. Inserm Unit 1110, Hôpitaux Universitaires de Strasbourg, 3 rue Koeberlé, F-67000 Strasbourg, France. \\ Email: Thomas.Baumert@unistra.fr; Daniel J. Felmlee, PhD. Plymouth University Peninsula Schools of Medicine \& Dentistry, The John Bull \\ Building, Research Way, Plymouth PL6 8BU, UK. Email: Daniel.Felmlee@plymouth.ac.uk.
}

Submitted May 21, 2016. Accepted for publication Jun 02, 2016.

doi: $10.21037 /$ tcr.2016.06.28

View this article at: http://dx.doi.org/10.21037/tcr.2016.06.28

The future of anti-cancer therapy is promising as immunotherapy strategies have been revolutionary in treatment of a number of cancers. However, some organs such as the liver are characterised by immunosuppression of $\mathrm{CD}^{+}$and $\mathrm{CD} 8^{+}$activity, necessitating alternative strategies for cancers in such tissues. This occurs at least partly through aberrant expression of alpha-fetoprotein that in turn dampens immune activation (1). Hepatocellular carcinoma (HCC) is the second leading cause of death from cancer worldwide (2). Complementary strategies to $\mathrm{CD}^{+}$and $\mathrm{CD} 8^{+} \mathrm{T}$ cell-mediated immunotherapies that specifically target HCC are needed for the future of effective and comprehensive cancer therapy. On the shortlist for a molecular target for such a therapy is CD147/ EMMPRIN.

CD147 is a transmembrane vesicular and cell-surface glycoprotein with two N-terminal extracellular-oriented immunoglobulin domains. CD147 was discovered as a factor produced from tumour cells that induced matrix metalloproteinases (MMPs) and collagenase activity from fibroblasts (3), thus gaining the moniker extracellular matrix metalloproteinase inducer (EMMPRIN) (4). While various tissues have detectable expression of CD147, a high percentage of malignant cancers overexpress the protein, including HCC $(5,6)$. The use of ${ }^{131} \mathrm{I}$ labelled CD147 specific humanized antibodies has been approved by the Chinese Food and Drug Administration under the drug name licartin or metuximab, and is entering Chinese clinics (7). Niu et al. were able to show that immunologically increasing the concentration of the radioisotope at tumor sites results in diminished tumor size and extended survival for treated rabbits relative to controls (8). Furthermore, individuals with advanced HCC who received licartin post-liver transplantation had less recurrence of HCC compared to placebo one year posttransplantation (26.7\% vs. $57.1 \%)(9)$.

Despite advances in using CD147 as a tumor targeting molecule, the biology of CD147 remains complex, with multiple regulating factors and pluripotent activities (Figure 1) $(4,6)$. The alias EMMPRIN indicates CD147's role in inducing fibroblasts to secrete MMPs, namely MMP-1, MMP-2, MMP-3, MMP-9, MT1-MMP, and MT2-MMP $(4,10)$. The stimulatory effect of these proteases could play a role in activating invasion and metastasis, one of the hallmarks of cancer (13). CD147 can homo-oligomerize and bind a number of binding partners such as cyclophilin A and monocarboxylate transporters (MCTs). Soluble cyclophilin A promotes migration and proliferation of multiple myeloma cells by binding to CD147, and plays a role in chemotaxis of myeloma cells from the blood to the bone marrow (14). CD147 complexes with MCTs to symport lactic acid and protons out of the cell, acidic products of the fermentative glycolysis produced by rapidly proliferating tumour cells (11). In the context of tumor growth, this function of CD147 allows rapid proliferation and metabolism by glycolysis, the "Warburg effect", without generating an acidic intracellular milieu. Over-expression of CD147 correlates with higher glucose uptake, lactate production, and diminished $\mathrm{p} 53$ expression relative to CD147 silenced cells $(12,15)$.

Rapidly proliferating tumor tissue requires energy consumption yielded by glycolysis and lactic acid 


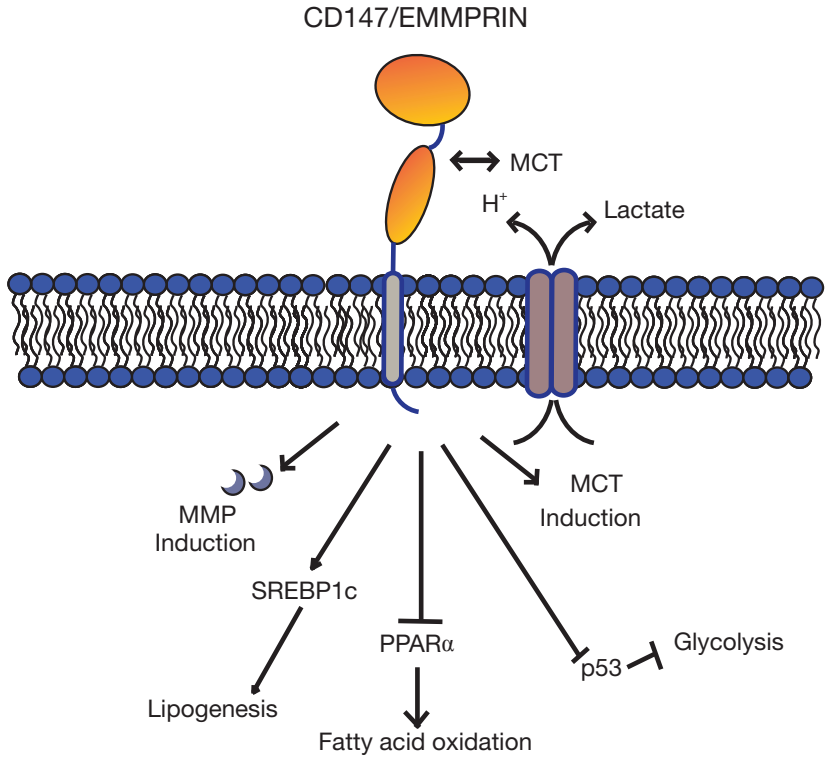

Figure 1 The multiple functions of CD147/EMMPRIN. CD147 was identified as a factor that induced matrix metalloproteinases (MMPs) to restructure the extracellular matrix (10). It also plays an important role in inducing monocarboxylate transporters (MCTs) and hetero-dimerizing with them to export damaging fermentative glycolysis products, lactate and protons (11). Investigators in Xi'an, China have identified that CD147 expression upregulates SREBP1c, and represses PPAR $\alpha$ and p53 $(7,12)$. The downstream effects result in increased lipogenesis, decreased fatty acid oxidation, and increased glycolysis. This metabolic profile suits rapidly proliferating tumors.

fermentation, and also needs production of cellular components for cell division, including a high demand for phospholipid synthesis. Since CD147 appears to act as a control switch for cells to enter anaerobic glycolysis, could this have knock-on effects into lipid metabolism? Li and colleagues utilized 4 public RNA-seq datasets of HCC tissues and found a correlation between CD147 expression and increased expression of genes involved in fatty acid metabolism (7). The authors then used two different HCC cell lines to down-regulate or knock out CD147 expression, along with restoring CD147 expression in the knocked-out line, to observe differences in fatty acid metabolism. CD147 expressing cells not only had higher levels of phospholipids but also had high triglyceride levels; similar to the lipid droplet accumulation observed in cancers previously (16). CD147 expression correlated with the lipogenesis promoting transcription factor SREBP1c, as well as downstream genes encoding lipogenic enzymes. Lipogenesis in HCC has been associated with the AKT-mTORC1 pathway (17), and analysis of these engineered cells showed that CD147 expression aligned with activation of this pathway. Similar lines of evidence showed correlations between diminished fatty acid oxidation genes through suppression of transcription factor PPAR $\alpha$ through MAPK signalling. The authors then implanted the CD147 knocked-out HCC cell lines, the cells with ectopically restored SREBP1c expression, or the cells with silenced PPAR $\alpha$ expression into the livers of nude mice. Both increased lipogenesis and diminished fatty acid oxidation separately could restore oncogenesis despite CD147 knockout, indicating that fatty acid metabolism is a key instrument in which CD147 is oncogenic.

CD147 is overexpressed in a number of tumor types and is a therapeutic target for directed immune-radiotherapy against HCC. Investigation of CD147 has revealed a number of different functions for the protein yet with ambiguous direct effects. Li et al. used genetic techniques to show that CD147 exerts tumorigenic activity by both upregulating lipogenesis and down-regulating fatty acid oxidation, thus meeting the phospholipid requirements for cell proliferation. The multiple roles played by CD147 appear related in that they all encourage rapid proliferation. These roles include inducing MMPs to restructure the extracellular matrix to make space for new cells, eschewing lactate to encourage rapid glycolysis and energy production, and now promoting lipogenesis to build membranes of daughter cells. The mystery that remains to be clarified is how does CD147, a surface transmembrane protein, play all of these roles. Since CD147 is a transmembrane protein with characteristic $\mathrm{N}$-terminal immunoglobulin domains, the effect is likely through signalling but there is a paucity of data describing intrinsic signalling motifs in CD147 (4). There is much cross-talk between glucose and lipid metabolism pathways and it may be difficult to precisely define the mechanism involving CD147's role in these processes. Investigations into this hub of so many oncogenic effects need to occur in order to improve our understanding of tumor development and treatment.

\section{Acknowledgements}

Funding: TF Baumert acknowledges grant support by the European Union (ERC-2008-AdG-HEPCENT, ERCAdG-2014-HEPCIR, FP7 HepaMab, and Interreg IV FEDER-Hepato-Regio-Net 2012), the Agence Nationale de Recherche sur le SIDA (ANRS) and the Direction Générale de l'Offre de Soins (A12027MS). 


\section{Footnote}

Provenance: This is a Guest Editorial commissioned by Section Editor An-Qiang Wang, MD (Department of Liver Surgery, Peking Union Medical College Hospital, Chinese Academy of Medical Sciences and Peking Union Medical College, Beijing, China).

Conflicts of Interest: The authors have no conflicts of interest to declare.

Comment on: Li J, Huang Q, Long X, et al. CD147 reprograms fatty acid metabolism in hepatocellular carcinoma cells through Akt/mTOR/SREBP1c and P38/PPAR $\alpha$ pathways. J Hepatol 2015;63:1378-89.

\section{References}

1. Ritter M, Ali MY, Grimm CF, et al. Immunoregulation of dendritic and $\mathrm{T}$ cells by alpha-fetoprotein in patients with hepatocellular carcinoma. J Hepatol 2004;41:999-1007.

2. Ferlay J, Soerjomataram I, Dikshit R, et al. Cancer incidence and mortality worldwide: sources, methods and major patterns in GLOBOCAN 2012. Int J Cancer 2015;136:E359-86.

3. Kataoka H, DeCastro R, Zucker S, et al. Tumor cellderived collagenase-stimulatory factor increases expression of interstitial collagenase, stromelysin, and $72-\mathrm{kDa}$ gelatinase. Cancer Res 1993;53:3154-8.

4. Grass GD, Toole BP. How, with whom and when: an overview of CD147-mediated regulatory networks influencing matrix metalloproteinase activity. Biosci Rep 2015;36:e0283.

5. Riethdorf S, Reimers N, Assmann V, et al. High incidence of EMMPRIN expression in human tumors. Int $\mathrm{J}$ Cancer 2006;119:1800-10.

6. Xiong L, Edwards CK 3rd, Zhou L. The biological function and clinical utilization of CD147 in human diseases: a review of the current scientific literature. Int J Mol Sci 2014;15:17411-41.

Cite this article as: Felmlee DJ, Baumert TF. CD147 handles lipid: a new role for anti-cancer target. Transl Cancer Res 2016;5(3):238-240. doi: 10.21037/tcr.2016.06.28
7. Li J, Huang Q, Long X, et al. CD147 reprograms fatty acid metabolism in hepatocellular carcinoma cells through Akt/mTOR/SREBP1c and P38/PPAR $\alpha$ pathways. J Hepatol 2015;63:1378-89.

8. Niu H, Wang R, Cheng J, et al. Treatment of (131)I-labeled anti-CD147 monoclonal antibody in VX2 carcinomainduced liver tumors. Oncol Rep 2013;30:246-52.

9. $\mathrm{Xu}$ J, Shen $\mathrm{ZY}$, Chen XG, et al. A randomized controlled trial of Licartin for preventing hepatoma recurrence after liver transplantation. Hepatology 2007;45:269-76.

10. Guo H, Zucker S, Gordon MK, et al. Stimulation of matrix metalloproteinase production by recombinant extracellular matrix metalloproteinase inducer from transfected Chinese hamster ovary cells. J Biol Chem 1997;272:24-7.

11. Hanahan D, Weinberg RA. Hallmarks of cancer: the next generation. Cell 2011;144:646-74.

12. Zhu D, Wang Z, Zhao JJ, et al. The Cyclophilin A-CD147 complex promotes the proliferation and homing of multiple myeloma cells. Nat Med 2015;21:572-80.

13. Le Floch R, Chiche J, Marchiq I, et al. CD147 subunit of lactate/H+ symporters MCT1 and hypoxia-inducible MCT4 is critical for energetics and growth of glycolytic tumors. Proc Natl Acad Sci U S A 2011;108:16663-8.

14. Huang Q, Li J, Xing J, et al. CD147 promotes reprogramming of glucose metabolism and cell proliferation in HCC cells by inhibiting the p53dependent signaling pathway. J Hepatol 2014;61:859-66.

15. Schneiderhan W, Scheler M, Holzmann KH, et al. CD147 silencing inhibits lactate transport and reduces malignant potential of pancreatic cancer cells in in vivo and in vitro models. Gut 2009;58:1391-8.

16. Currie E, Schulze A, Zechner R, et al. Cellular fatty acid metabolism and cancer. Cell Metab 2013;18:153-61.

17. Calvisi DF, Wang C, Ho C, et al. Increased lipogenesis, induced by AKT-mTORC1-RPS6 signaling, promotes development of human hepatocellular carcinoma. Gastroenterology 2011;140:1071-83. 